\title{
Statistical Transform of Signal Field with ASE Noise through a Fiber Amplifier
}

\author{
Jing Huang, Jianquan Yao \\ ${ }^{1}$ Physics Department, South China University of Technology, Guangzhou, China \\ ${ }^{2}$ College of Precision Instrument and Opto-Electronics Engineering, Tianjin University, Tianjin, China \\ Email: huanggesheng@tom.com,jqyao@tju.edu.cn
}

Received 25 May 2016; accepted 18 August 2016; published 25 August 2016

\begin{abstract}
While the signal field + ASE noise pass through a span of transmission fiber, a dispersion compensation grating and a fiber amplifier(with the generation of ASE noise), the nonlinear Fokker-Plank equations, describing the probability transforms of the field, are established and solved. Based on these statistical theories, the probability distributions of the signal + ASE noise field through $50 \mathrm{~km}$ NZDSF, a dispersion compensation grating and a fiber amplifier link, are obtained. The dispersion and nonlinear effects in transmission fiber induce frequency offsets in the probability distribution of field and they cannot be dissipated by dispersion compensation. The generation of ASE noise in the amplifier will accelerate this frequency offset.
\end{abstract}

\section{Keywords}

Probability Density Function, Frequency Offset, Nonlinear Fokker-Plank Equation

\section{Introduction}

The statistical model of phase noise induced by the interplay between amplifier spontaneous emission (ASE) noise and fiber Kerr nonlinearity, is extensively studied during the last decade. The probability density function (p.d.f.) of ASE noise + signal field is necessary to analyze the noise properties and evaluate the system performances.

By converting a Cartesian into a polar description, the nonlinear phase noise (NPN) (the additive component of ASE noise) was identified to be nearly Gaussian [1]. Then in [2], by linearizing the interaction between a signal and noise in the limit of a distributed system, authors evaluated the closed Gaussian form ASE power spectral density. Even if the received ASE was non-stationary in time due to pulse shape and modulation, they showed that it could be approximated by an equivalent stationary process, as if the signal was continuous wave (CW). Such a method avoided the calculation of nonlinear phase statistics. The CW-equivalent ASE model was also used to evaluate bit-error-rate by an extension of the known Karhunen-Loéve method. Additionally, the combined regular-logarithmic perturbation model was used to derive the basic propagation equations of ASE noise in amplified multi-span optical systems [3]. Also, in situations that no analytical theory was available for the dispersion and nonlinearity managed transmissions, the important sample algorithms performed a direct computation of the random optical soliton phase statistical distribution under the action of nonlinear phase noise 


\section{[4].}

But by the experimental observation and performance study of the differential phase-shift-keying transmission systems [5] [6], the results showed that the p.d.f. of nonlinear phase noise deviated from the Gaussian distribution, and this characteristic negated the benefit of a balanced receiver [7]. Thus, the calculation of nonlinear phase noise had to turn back to the original theory [8] and this distributed model was described as the transform of stochastic Wiener process [9]. Firstly, it was given as a summation from the contribution of many fiber spans. If the number of fiber spans was very large, the summation was replaced by an integration. Several years ago, an approximate but analytical treatment of the statistical properties of the NPN of an isolated RZ pulse was presented and it revealed that the variance of NPN in a dispersion-managed fiber-optics link might be reduced by properly decreasing the duty cycle of an RZ pulse [10].

Taking the dispersion effect into account, the p.d.f. of signal pulse with nonlinear phase noise was broadened and the broadening was asymmetrical with respect to the mean nonlinear phase shift [11]. Based on the assumption that phase noise was linearly approximated, T. Pollet considered the BER performance in OFDM system in the presence of phase noise and frequency offset [12]. The nonlinear stochastic differential equation was also developed to approximate phase noise in oscillators. E. Costa and S. Pupolin studied M-QAM-OFDM system performance in the presence of a nonlinear amplifier and phase noise [13]. They approximated the phase noise linearly. A more detailed analysis was still required to study phase noise's influence. So in [14], a nonlinear approximation about phase noise including the second order term of phase noise was proposed.

In this paper, the probability transform is studied when ASE noise +signal field pass through a span of transmission fiber and a fiber amplifier where ASE noise is generated and described by a delta $(\delta)$-function. The dispersion effect of fiber is taken into account, so the statistical properties of the field are nonstationary. In the amplifier, ASE noise is generated, and thus based on the birth theory of stochastic, the field's statistical transform is established and the probability distribution through $50 \mathrm{~km}$ nonzero-dispersion-shifted fiber (NZDSF), a dispersion compensation grating and an erbium-doped fiber amplifier (EDFA), are obtained.

\section{Theory}

The optical field envelope in an amplified transmission system is governed by the nonlinear Schrodinger(NLS) equation [15]

$$
i \frac{\partial u}{\partial z}-\frac{\beta_{2}}{2} \frac{\partial^{2} u}{\partial t^{2}}=-\gamma \exp [-w(z)]|u|^{2} u+i R(z, t) \delta(z-m L)
$$

where $\beta_{2}$ is the dispersion profile, $\gamma$ is the nonlinear coefficient, $w(z)=\int_{0}^{z} a(s) d s$ and $a(z)$ is the fiber loss profile. $R(z, t)=\sum_{m=1}^{N} n^{(m)}(t)$ represents the noise field due to an amplification. $L_{m}$ is the amplifier location. $N$ is the number of amplifiers and $n^{(m)}(t)$ is the noise field due to the amplifier located at $L_{m}$. The mean and autocorrelation functions of the noise field are given by

$$
<n^{(m)}(t)>=0, \quad\left\langle n^{(m)}(t) n^{(m) *}\left(t^{\prime}\right)>=n_{s p} h \bar{v}\left(G_{m}-1\right) \delta\left(t-t^{\prime}\right) .\right.
$$

where $G_{m}$ is the gain of the amplifier, $n_{s p}$ is the spontaneous noise factor, $h$ is the Plank's constant and $\bar{v}$ is the mean optical carrier frequency.

Disregard the stochastic item in (1), we can calculate the optical field without ASE noise item by the split-step method [16]

$$
v(z, t)=\exp [d z(\hat{D}+\hat{N})] v(z-d z, t)
$$

Now, (1) can be written as

$$
\frac{\partial u}{\partial z}=v(z, t)+R(z) \delta(z-m L)
$$
[17]

Assume $f(u)$ is the arbitrary function of the signal + noise field and $u$ has a probability density $p(u)$ 


$$
\begin{aligned}
& d f(u)=f(u+d u)-f(u)=f^{\prime}(u) d u+1 / 2 f^{\prime \prime}(u)(d u)^{2}+\cdots \\
& \approx f^{\prime}(u)[v(z, t) d z+d W(z, t)]+1 / 2 f^{\prime \prime}(u) d z+\cdots
\end{aligned}
$$

where $W(z, t)$ refers to the Weiner process.

$$
\begin{aligned}
& \int d u \partial_{z} f(u) p(u)=f(u) p(u) \frac{\partial u}{\partial z}-\int d u f(u) \partial_{z} p(u) \\
& \approx \int d u p(u)\left\{f^{\prime}(u)[v(z, t)+R(z) \delta(z-m L)]+1 / 2 f^{\prime \prime}(u)\right\} \\
& =f(u) p(u) \frac{\partial u}{\partial z}-\int d u f(u)\left\{\partial_{u}[v(z, t) p(u)]-1 / 2 \partial_{u}^{2}[p(u)]+\rho_{m} p(u)\right\}
\end{aligned}
$$

According to the property of $\delta^{\prime}(z-m L)$

$$
\rho_{m}=R(z) \delta(z-m L) / z
$$

we get the generalized Fokker-Plank equation describing the signal field with ASE noise

$$
\partial_{z} p(u)=\partial_{u}[v(z, t) p(u)]-\frac{1}{2} \partial_{u}^{2}[p(u)]+\rho_{m} p(u)
$$

In particular, the isolated system ( $\rho_{m}=0$ and $\partial_{z} p(u)=0$ ) produces a stationary solution in the form [17]

$$
p_{0}(u)=c^{\prime} \exp \left[2 \int_{-\infty}^{u} v(z, t) d u\right]
$$

We assume the transient solution (only $\rho_{m}=0$ ) is the form

$$
\begin{gathered}
p(u, z)=\sum_{n=0}^{\infty} T_{n} e^{-\lambda_{n} z} \varphi_{n}(u) \\
\hat{L}_{F P}\left[\varphi_{n}(u)\right]=\frac{d}{d u}\left[v(z, t) \varphi_{n}(u)\right]-\frac{d^{2}}{2 d u^{2}}\left[\varphi_{n}(u)\right]=-\lambda_{n} \varphi_{n}(u) \\
\varphi_{n}=\frac{1}{\sqrt{\sqrt{\pi} 2^{n} n !}}(-1)^{n} e^{u^{2}} \frac{d^{n}}{d u^{n}} e^{-u^{2}} \\
\lambda_{n}=n-1 \\
\int_{-\infty}^{+\infty} \varphi_{n} \varphi_{l} e^{-u^{2}} d u=\delta_{n l}= \begin{cases}1 & \text { if } l=n \\
0 & \text { if } l \neq n\end{cases}
\end{gathered}
$$

$T_{n}$ is determined by the launched pulse and $\lambda_{0}=0$, so $\varphi_{0}=p_{0}(u)$.

Taking the ASE noise as a perturbation item, we now try to solve the perturbed Fokker-Planck Equation (8) [8] [18]

$$
\begin{gathered}
\hat{O}[\psi(u)]=\hat{L}_{F P}[\psi(u)]+\rho_{m} \psi(u) \\
\psi(u)=\sum_{n=1}^{\infty} Y_{n} e^{\Lambda_{n} z} \Lambda_{n} \psi_{n}
\end{gathered}
$$

Its eigenvalue and eigenfunction can be expanded as

$$
\begin{gathered}
\Lambda_{n}=\lambda_{n}+\kappa \lambda_{n}^{(1)}+\kappa^{2} \lambda_{n}^{(2)}+\cdots=\lambda_{n}+\sum_{l=1} \kappa^{l} \lambda_{n}^{(l)} \\
\psi_{n}(u)=\varphi_{n}(u)+\sum_{l=1} \kappa^{l} \varphi_{n}^{(l)}(u)
\end{gathered}
$$

Finally, comparing the terms $\kappa, \kappa^{2}, \cdots$ in both sides, we get

$$
\hat{L}_{F P}\left[\varphi_{n}^{(1)}(u)\right]+\lambda_{n} \varphi_{n}^{(1)}(u)=-\left(\frac{\rho_{m}}{\kappa}+\lambda_{n}^{(1)}\right) \varphi_{n}(u)
$$




$$
\hat{L}_{F P}\left[\varphi_{n}^{(l)}(u)\right]+\frac{\rho_{m}}{\kappa} \varphi_{n}^{(l-1)}(u)=-\sum_{k+p=l} \lambda_{n}^{(k)} \varphi_{n}^{(p)}(u)
$$

(17) and (18) can be written as

$$
\begin{gathered}
\hat{L}_{F P}\left[\varphi_{n}^{(l)}(u)\right]+\lambda_{n}^{(l)} \varphi_{n}^{(l)}(u)=-\frac{\rho_{m}}{\kappa} \varphi_{n}^{(l-1)}(u)-\sum_{k+p=l-1} \lambda_{n}^{(k)} \varphi_{n}^{(p)}(u)=\zeta(u) \\
\varphi_{n}^{(l)}(u)=\int_{-\infty}^{+\infty} G\left(u, u^{\prime}\right) \zeta\left(u^{\prime}\right) d u^{\prime} \\
G_{p}\left(u, u^{\prime}\right)=\frac{\varphi_{p}\left(u^{\prime}\right) \varphi_{p^{\prime}}(u)-\varphi_{p}(u) \varphi_{p^{\prime}}\left(u^{\prime}\right)}{\left|\begin{array}{ll}
\varphi_{p}(u) & \varphi_{p^{\prime}}\left(u^{\prime}\right) \\
\varphi_{p}^{\prime}(u) & \varphi_{p^{\prime}}^{\prime}\left(u^{\prime}\right)
\end{array}\right|}\left(p \neq p^{\prime}\right) \\
\varphi_{n}^{(l)}(u)=\sum_{p=1}^{\infty} b_{p} \int_{-\infty}^{+\infty} G_{p}\left(u, u^{\prime}\right) \zeta\left(u^{\prime}\right) d u^{\prime}
\end{gathered}
$$

The item of $p$ in (23) is definite because of the orthogonality and normalization of (13). Also, the coefficients $b_{p}$ and $Y_{n}$ are determined by the input field of the amplifier. Note that, this model requires that the first order differential of $\varphi_{n}(u)$ is continuity, otherwise, the Green function of Equation (22) has no meaning.

\section{Discussion}

In this section, we will simulate the p.d.f. of the ASE noise+ signal field after transmitted in NZDSF, grating and an amplifier link such as Figure 1. The measured points $a, b$ and $c$ correspond to the sub-figures $a, b$ and $c$ in Figure 2, respectively.

Fibers parameters are: $a=0.21(\mathrm{~dB} / \mathrm{km}), \gamma=2.2(/ \mathrm{km} / \mathrm{W}), D=4.4(\mathrm{ps} / \mathrm{nm} / \mathrm{km})$. The pulse is:

$$
u(0, t)=u_{0} \exp \left(-t^{2} / 2 / t_{0}^{2}\right)
$$

$u_{0}=5 \mathrm{~mW}, L=50 \mathrm{~km}$ and $t_{0}=25 / 3 \mathrm{ps}, t_{0}$ is the half-width at $1 / e$ intensity point.

There are frequency offsets in the p.d.f. when the dispersion and nonlinear effects in transmission fiber are taken into account and they cannot be dissipated by dispersion compensation. Figure 2(a) and Figure 2(b) present these properties. The probability distribution after NZDSF is based on (9) and (10) and that after the amplifier is according to (20) and (21) (with ASE noise item). The probability transform passing through the dispersion compensation grating is from [19] by the matrix

$$
h=\left|\begin{array}{cc}
\cos \left(\frac{D}{2} \frac{\partial^{2}}{\partial t^{2}}\right) & -\sin \left(\frac{D}{2} \frac{\partial^{2}}{\partial t^{2}}\right) \\
\sin \left(\frac{D}{2} \frac{\partial^{2}}{\partial t^{2}}\right) & \cos \left(\frac{D}{2} \frac{\partial^{2}}{\partial t^{2}}\right)
\end{array}\right|
$$

where $D$ is the compensation dispersion supplied by grating.

The sideband induced by dispersion and nonlinear effects is not transient and it exists at a certain probability. From the mathematical formula, the solution (11) is the Hermite polynomial and it is a series of vibration functions. When they add together several times, the side bands occur but still with a lot of chances, they are located neither in the field nor in the sideband regions. These are determined by the coefficient $v(z, t)$ (the ASE noise

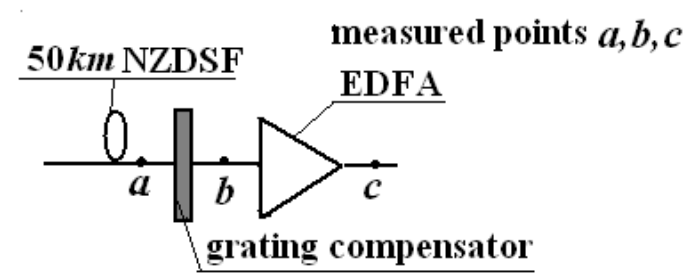

Figure 1. Schematic of the simulation setup. 
+ signal field) and similar to the case of [18] by mixing the Gauss p.d.f. The amplifier, amplifying the signal and generating ASE noise, will result in relative decrease of the non-field and non-sideband probability but it can't change the impact of dispersion and nonlinear effects on field statistical distribution (Figure 1(c)).

Phase shifts caused by the nonlinear effect are also consistent with [20] and as its authors had expected that, if the dispersion effect was taken into account, there were the asymmetric modulation side-bands occurring.

It is most likely that this figure can't clearly show the impact of local ASE noise (generated in EDFA). So, in Figure 3, we extract the distribution brought by the generated ASE noise.

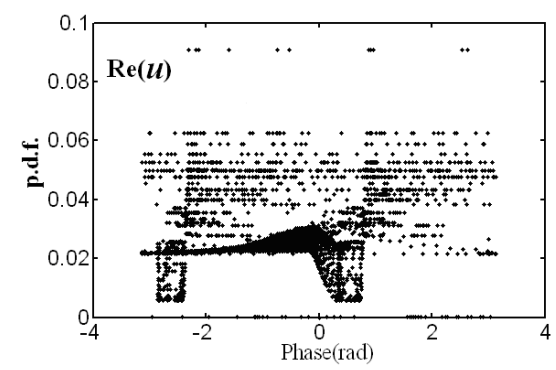

(a)

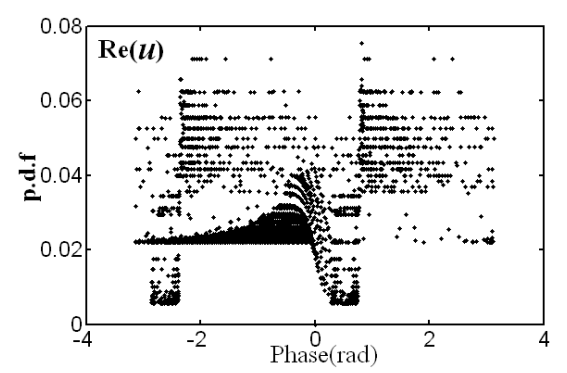

(c)

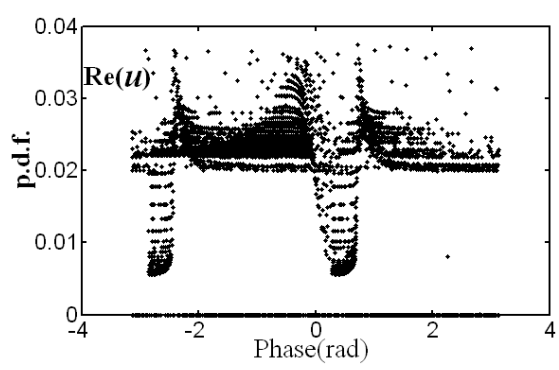

(e)

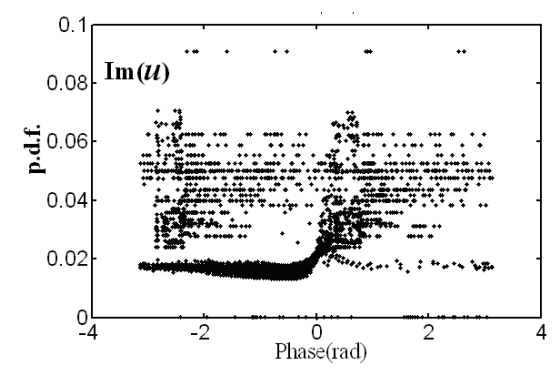

(b)

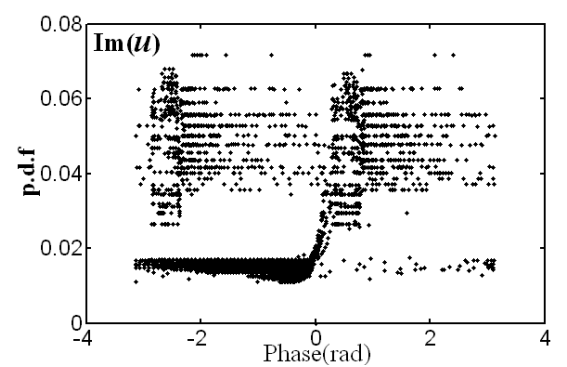

(d)

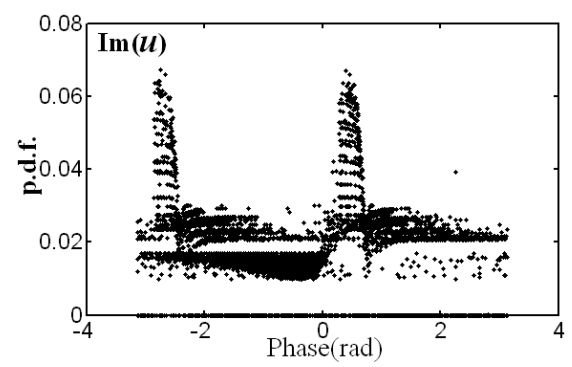

(f)

Figure 2. The probability distributions of field in the measured points $a, b$ and $c$. (a) after $50 \mathrm{~km}$ NZDSF; (b) after 50 km NZDSF + a grating; (c) after 50 km NZDSF + agrating + an amplifier.

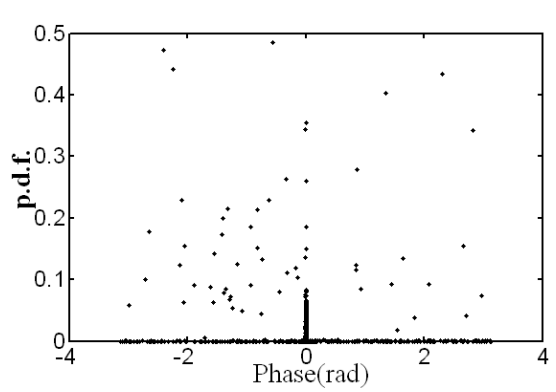

(a)

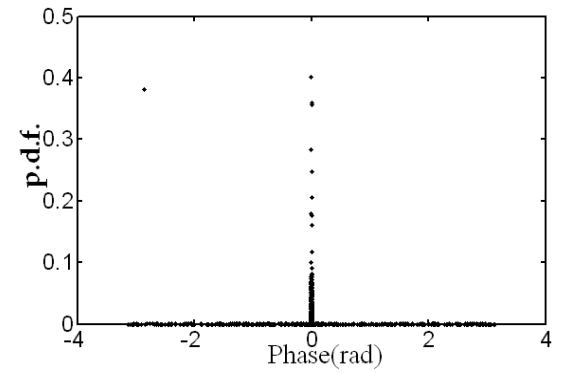

(b)

Figure 3. The probability distribution (a) real value; (b) image value brought by the generated. ASE noise at the wavelength of signal. 


\section{Conclusion}

Therefore, in the fiber + dispersion grating + amplifier transmission systems, the evaluation about the statistical transform of signal + ASE noise field shows that the frequency offset of the field's probability distribution induced by dispersion and nonlinear effects in transmission fiber cannot be dissipated by dispersion compensation and the ASE noise generated in the amplifier is a perturbation item and has weak impact on the field's p.d.f., but it accelerates the field's frequency offset.

\section{References}

[1] Mecozzi, A. (1994) Long-Distance Transmission at Zero Dispersion Combined Effect of the Kerr Nonlinearity and the Noise of the In-Line Amplifiers. J. Opt. Soc. Am. B, 11, 462-469. http://dx.doi.org/10.1364/JOSAB.11.000462

[2] Serena, P., Orlandini, A. and Bononi, A. (2006) Parametric-Gain Approach to the Analysis of Single-Channel DPSK/ DQPSK Systems with Nonlinear Phase Noise. IEEE J. Lightwave Technol., 24, 2026-2037. http://dx.doi.org/10.1109/JLT.2006.872686

[3] Secondini, M., Forestieri, E. and Menyuk, C.R. (2009) A Combined Regular-Logarithmic Perturbation Method for Signal-Noise Interaction in Amplified Optical Systems. IEEE J. Lightwave Technol., 27, 3358-3369. http://dx.doi.org/10.1109/JLT.2009.2012873

[4] Tonello, A., Wabnitz, S., Gabitov, I. and Indik, R. (2006) Importance Sampling of Gordon-Mollenauersoliton Phase Noise in Optical Fibers. IEEE Photon. Technol. Lett., 18, 886-888. http://dx.doi.org/10.1109/LPT.2006.871817

[5] Wei, X. and Liu, X. (2003) Analysis of Intrachannel Four-Wave Mixingin Differential Phase-Shift Keying Transmission with Large Dispersion. Opt. Lett., 28, 2300-2302. http://dx.doi.org/10.1364/OL.28.002300

[6] Kim, H. and Gnauck, A.H. (2003) Experimental Investigation of the Performance Limitation of DPSK Systems Due to Nonlinear Phase Noise. IEEE Photon. Technol. Lett., 15, 320-322. http://dx.doi.org/10.1109/LPT.2002.807921

[7] Ho, K.-P. (2003) Probability Density of Nonlinear Phase Noise. J. Opt. Soc. Am. B, 20, 1875-1879. http://dx.doi.org/10.1364/JOSAB.20.001875

[8] Papoulis, A. (1984) Probability, Random Variables, and Stochastic Processes. McGraw Hill, New York.

[9] Mecozzi, A. (2004) Probability Density Functions of the Nonlinear Phase Noise. Opt. Lett., 29, 673-675. http://dx.doi.org/10.1364/OL.29.000673

[10] Tonello, A., Wabnitz, S. and Boyraz, O. (2005) Duty Ratio Control of Nonlinear Phase Noise in Dispersion Managed WDM Systems Using RZ-DPSK Modulation. Optical Fiber Commun. Conf. (OFC), Anaheim.

[11] Vanin, E., Jacobsen, G. and Berntson, A. (2007) Nonlinear Phase Noise Separation Method for On-Off Keying Transmission System Modeling with Non-Gaussian Noise Generation in Optical Fibers. Opt. Lett., 32, 1740-1742.

[12] Pollet, T., van Bladel, M. and Moeneclaey, M. (1995) BER Sensitivity of OFDM Systems to Carrier Frequency Offset and Wiener Phase Noise. IEEE Transactions on Communication, 43, 191-193.

[13] Costa, E. and Pupolin, S. (2002) M-QAM-OFDM System Performance in the Presence of a Nonlinear Amplifier and Phase Noise. IEEE Transactions on Communications, 50, 462-472. http://dx.doi.org/10.1109/26.990908

[14] Ryu, H.-G., Li, Y.S. and Park, J.-S. (2004) Nonlinear Analysis of the Phase Noise in the OFDM Communication System. IEEE Transactions on Communications, 50, 54-63.

[15] Kumar, S. (2005) Effect of Dispersion on Nonlinear Phase Noise in Optical Transmission Systems. Opt. Lett., 320, 3278-3280. http://dx.doi.org/10.1364/ol.30.003278

[16] Agrawal, G.P. (2001) Nonlinear Fiber Optics. 3rd Edition, Academic Press.

[17] Gardiner, C.W. (1983) Handbook of Stochastic Method for Physics, Chemistry and the natural Sciences. SpringerVerlag, Berlin Heidelberg, New York, Tokyo. http://dx.doi.org/10.1007/978-3-662-02377-8

[18] Mukherjee, A. and Sengupta, A. (2010) Estimating the Probability Density Function of a Nonstationary Non-Gaussian Noise. IEEE Transactions on Industrial Electronics, 57, 1429-1435. http://dx.doi.org/10.1109/TIE.2009.2039451

[19] Wang, J. and Petermann, K. (1992) Small Signal Analysis for Dispersive Optical Fiber Communication Systems. IEEE J. Lightwave Technol., 10, 96-100. http://dx.doi.org/10.1109/50.108743

[20] Dlubek, M.P., Phillips, A.J. and Larkins, E.C. (2008) Nonlinear Evolution of Gaussian ASE Noise in ZMNL Fiber. IEEE J. Lightwave Technol., 26, 891-898. http://dx.doi.org/10.1109/JLT.2008.917373 


\section{Submit or recommend next manuscript to SCIRP and we will provide best service for you:}

Accepting pre-submission inquiries through Email, Facebook, LinkedIn, Twitter, etc.

A wide selection of journals (inclusive of 9 subjects, more than 200 journals)

Providing 24-hour high-quality service

User-friendly online submission system

Fair and swift peer-review system

Efficient typesetting and proofreading procedure

Display of the result of downloads and visits, as well as the number of cited articles

Maximum dissemination of your research work

Submit your manuscript at: http://papersubmission.scirp.org/ 